\title{
Phenotypic and Genotypic Characterization and the Study of Type III Secretion System of Pseudomonas aeruginosa from Surat Region
}

\author{
Priyanka Gajjar ${ }^{1}$, Manisha Shah ${ }^{2}$ \\ Department of Microbiology, Shree Ramakrishna Institute of Computer Education and Applied Sciences, Surat, Gujarat, India
}

\begin{abstract}
Aim \& Objectives: The present study was aimed to determine phenotypic characterization of virulent Pseudomonas aeruginosa and its Type 3 Secretion system from Surat region. Methods: 125 clinical Pseudomonas aeruginosa were procured from different laboratories of Surat and 53 environment Pseudomonas aeruginosa isolated from 125 various environment and vegetable samples. Antimicrobial susceptibility testing was performed by Kirby Bauer Disc Diffusion method. The phenotypic screening evaluated for six soluble virulence factors (amylase, Caseinase, gelatinase, haemolysins, lecithinase, and lipase). Biofilm formation was detected by Tube method and Congo red agar method. Four virulence genes (exoS, exoT, exoU, exoY) were screened in selected isolates by PCR. Results: A high percentage of resistance rates $(89.60 \%$ to amoxyclav and $88 \%$ to Cefuroxime) were observed amongst most of the isolates. Soluble enzymes were expressed in variable proportions (Gelatinase $96.80 \%$, Lipase 96\%, Lecithinase 88\%, and Caseinase $88 \%$ ). Tube method was considered to be superior to congo red agar method. The most frequent virulent gene detected was exoU (100\%). Conclusions: Clinical and environmental virulent Pseudomonas aeruginosa harbour various genes that represent type 3 secretary systems and have high level of resistance that may result in to therapeutic failure.
\end{abstract}

Keywords: Pseudomonas aeruginosa Type III secretion system Virulence factors

\section{Introduction}

Pseudomonas aeruginosa is an opportunistic pathogen that causes a wide range of acute infections in hospitalized and immuno- compromised patients. The pathogenicity of Pseudomonas aeruginosa is attributed because of its numerous secreted virulence factors [Shaver and Hauser, 2004; Galan and Collmer, 1999; Wong et al., 2008]. Many of the organisms related to food plants are non- pathogenic, including vegetables that might be contaminated through improperly- treated water and fertilizers or may be compromised by the use of biocides during cultivation [Deep et al., 2011 and Bjarcholt, 2013]. The number and wide range of virulence factors possessed by this bacterium includes; Pili, lipopolysaccharides, flagella, alkaline protease, siderophores and type III secretion system [Todar, 2009]. Among all of these T3SS is considered as an important factor contributed to the cytotoxicity and invasion processes [Shaver and Hauser, 2004; Galan and Collmer, 1999; Wong et al., 2008]. T3SS allows bacterium to directly inject their effector proteins in to host cells [Veesenmeyer et al., 2009]. Till date four effector proteins have been identified: ExoU, a phospholipase; ExoY, an adenylate cyclase, and ExoS \& ExoT, bifunctional proteins [Hyun et al., 2014]. The first identified T3SS toxins exoT and exoS, share $75 \%$ homology at amino acid level [Engel, 2003]. ExoS is the major cytotoxin involved in colonization, invasion and dissemination during infection [Lee et al., 2005]. It targets ras- like proteins, inhibits bacterial internalization by eukaryotic cells, DNA synthesis and induces apoptosis [Engel, 2003 and Vance et al., 2005]. ExoT targets host- kinases involved for adhesion and phagocytosis [Vance et al., 2005]. ExoY is an adenylate cyclase, which cleaves the intracellular cAMP that causes rounding of certain cell types [Lin et al., 2006].The most recently identified T3SS toxin, exoU shows marked cytotoxic effects [Vance et al., 2005 and Lin et al., 2006].
The intrinsic and acquired resistance of Pseudomonas aeruginosa to antibiotics is due to active efflux systems, reduced cell wall permeability, plasmid acquisition, and enzyme expression or by biofilm formation [Rasamiravaka et al., 2015].

The purpose of this study was to analyse the phenotypic nd genotypic characteristics and type III secretion system of Pseudomonas aeruginosa isolates from clinical and environmental sources.

\section{Literature Survey}

Pseudomonas aeruginosa is a Gram negative, aerobic, rod shaped bacterium, motile by polar flagella [Ryan et al., 2004]. Growth occurs at a wide range of temperatures, 6$42^{\circ} \mathrm{C}$, and the optimum temperature is $37^{\circ} \mathrm{C}$. It grows on MacConkey, forming non-lactose fermenting colonies [Pitt, 1996]. Pseudomonas aeruginosa produces a water soluble pigment called pyocyanin and pyoverdin [Baron et al., 1981 and Meyer et al., 1978]. It colonizes human body sites such as perineum, axilla, ear, nasal mucosa and throat. Pseudomonas aeruginosa is known as an opportunistic pathogen that rarely causes disease in healthy subjects. This bacterium causes disease by disrupting the physical barriers or by- passing from surgical devices and dysfunction of the immune defence mechanisms, as a result it is also known as nosocomial pathogen.

According to data of the Centre for Disease Control and Prevention National Nosocomial Infection Surveillance System, in the USA, Pseudomonas aeruginosa was the second most common cause of nosocomial pneumonia, the third most common cause of nosocomial urinary tract infections, and the seventh most common cause of nosocomial bacteraemia [NNIS, 1999]. In Europe, this organism was found to be the third most common to cause 


\section{International Journal of Science and Research (IJSR) \\ ISSN (Online): 2319-7064}

Index Copernicus Value (2015): 78.96 | Impact Factor (2015): 6.391

nosocomial infections in intensive care units [Vincent et al., 1995]. Approximately $40 \%$ to more than $60 \%$ mortality rates have been reported in bacteremic nosocomial pneumonia and in ventilator associated pneumonia [Crouch et al., 1996, Mayhall, 1997, Rello, 1997].

Pseudomonas aeruginosa causes urinary tract infections related to catheterization or other invasive procedure that may lead to bacteraemia [Pollack, 2000]. Wound infections related to burn patients often leads to bacteraemia. Bacteraemia and septic shock are mostly observed in immuno-compromised patients that are associated with high mortality rates (from one-third to almost two-thirds of cases) [Bodey et al., 1985, Gallagher, 1989, Siegman et al., 1998, Collin et al., 2001]. Different conditions such as severe neutropenia, mucosal ulcers, and malignancies lead to a risk for bacteraemia [Pollack, 2000, Aquino et al 1995, Fishman et al., 1998, Pizzo et al., 1999]. Pseudomonas aeruginosa is most commonly found in cystic fibrosis patients. The abnormal airway epithelia of these patients allow long-term colonization by this bacterium and, once they get infected, they rarely fade away and lead to chronic lung diseases [Pollack, 2000, and Davies, 2002].

Pseudomonas aeruginosa possess complex virulence factors. Pilli and other adhesions are important for the colonization of mucous membranes and other surfaces [Prince, 1992]. The production of a mucoid exo-polysaccharide matrix (lipopolysaccharide, LPS) is important for growth as a biofilm, which is responsible for escaping from host immune responses and protects it against antimicrobial agents [Boyd et al., 1995 and Drenkard, 2002].

Pseudomonas aeruginosa is the only Gram negative microbe capable of producing the distinctive water-soluble pigment pyocyanin [Alberto, 1981]. This bacterium secrets different extracellular products like elastase, alkaline protease, cytotoxin, phospholipase $\mathrm{C}$ and rhamnolipids, which are responsible for pathogenesis. Several proteolytic enzymes produced by this bacterium degrade a wide range of substrates including casein, elastin, gelatine, collagen and fibrin [Nicas, 1986]. Pseudomonas aeruginosa produces two different haemolysins, (i) a heat-labile enzyme, phospholipase C (Esselman 1961) and (ii) a heat-stable rhamnolipid (Sierra et al., 1960). Phospho-lipase C hydrolyzes phosphatidylcholine in erythrocyte membrane to phosphorylcholine and diacylglycerol and probably acts with an alkaline phosphatase to cleave inorganic phosphate from phospholipids. It also produces lipolytic enzymes that degrade tween 20 or 80 [Steur et al., 1986]. Exotoxin A produced by Pseudomonas aeruginosa catalyzes ADP ribosylation and inactivation of elongation factor 2 that leads to inhibition of protein biosynthesis and cell death [Wick et al., 1990].

Pseudomonas aeruginosa has become resistant to a variety of antimicrobial agents such as $\beta$-lactams, aminoglycosides, chloramphenicol, quinolones, tetracyclines and sulphonamides due to widespread use of antibiotics and advancements in medical and surgical technology (Livermore, 2002 and Nordmann, 2002). It acquires resistant to antimicrobial agents via the alteration of target site, production of $\beta$-lactamases; intrinsic drug resistance, which includes low membrane permeability and an active efflux system. $\beta$ - lactamases are enzymes that confer antibiotic resistance to their hosts by hydrolysis of the amide bond of the four-membered $\beta$-lactam ring [Wilke et al., 2005].

Multidrug-resistance efflux pumps of Pseudomonas aeruginosa are either chromosomally encoded or plasmid encoded [Akama et al., 2005]. They belong to five families of transporters namely; the major facilitator super-family (MFS), the adenosine triphosphate (ATP)-binding cassette (ABC) super-family, the small multidrug-resistance (SMR) family and the resistance nodulation-cell division (RND) super-family and the multidrug and toxic compound extrusion (MATE) family [Kumar et al., 2005]. Resistance to aminoglycosides is acquired by the production of aminoglycoside-modifying enzymes encoded by horizontally acquired resistance determinants, or by mutations [Hancock, 1998].

T3SS is the major virulence factor that contributes its cytotoxicity and acute infections [Hauser et al., 2002]. The T3SS of Pseudomonas aeruginosa was discovered in 1996 [Yahr et al., 1996] and 36 genes are associated with the function of T3SS [Frank, 1997 and Brutinel et al., 2008]. The gene encoding elements of machinery and gene encoding regulatory proteins are organized in operons. This machinery is made up of 20 different proteins and is called Psc, Pop or Pcr components [Filloux, 2011]. In Pseudomonas aeruginosa four effectors have been identified namely; ExoS, ExoT, ExoU, ExoY. ExoS and ExoT possess GTPase activating protein domain and adenosine diphosphate ribosyl transferase domain [Fleizig, 1997]. ExoY has an adenylate cyclase activity. ExoU is the most potent having phospholipase activity [Sato and Frank 2004]. These four effector proteins are transported through needle like structure in unfolded state and targeted to the T3SS by chaperone to which they bind prior to secretion [Persot et al., 2003].

\section{Methodology}

\section{Sample collection and transportation:}

The laboratory work was carried out from December, 2014 to May, 2017. 125 Different clinical samples were procured from different laboratories of Surat. 125 environment and vegetable samples were collected from vendors and environment from Surat, Gujarat, India. All Environment and vegetable samples were collected by a sterile cotton swab in a closed swab tube. Samples were collected and transported at room temperature or refrigerate after collection. Specimens must be tested within 72 hours after collection. [Konemann and Roberts, 1985].

\section{Isolation and Identification of Pseudomonas aeruginosa:}

A total of 125 clinical samples were collected from different laboratories of Surat and were reconfirmed as Pseudomonas aeruginosa by standard microbiological procedures. A total of 53 isolates from 125 environment and clinical samples were confirmed as Pseudomonas aeruginosa. All isolates were identified by colony characteristics and growth on microbiological media that includes lactose non- fermenter colonies on MacConkey's agar, Pigment production on

\section{Volume 6 Issue 7, July 2017 www.ijsr.net}




\section{International Journal of Science and Research (IJSR) \\ ISSN (Online): 2319-7064}

Index Copernicus Value (2015): 78.96 | Impact Factor (2015): 6.391

Kings B Medium and Oxidase and catalase tests positive [Bergey's manual of Determinative Bacteriology, $9^{\text {th }}$ edition, and Jean F. Macfaddin, Biochemical tests for Identification of Medical Bacteria, $3^{\text {rd }}$ edition].

\section{Antibiotic Susceptibility Testing:}

Antibiotic Susceptibility Testing was performed by the standard CLSI disc diffusion method using following antibiotics; Gentamicin, Netilin, Amikacin, Amoxyclav, Imipenem, Meropenem, Levofloxacin, Tobramicin, Cefuroxime, Piperacillin/ Tazobatam, CIT, Cefepime. Inocula were prepared by standard MacFarland concentration. Mueller Hinton plated were incubated at 37C for 16 to 18 hours after inoculation with organisms and placement of the disks, and zones of inhibition were measured [CLSI Document M02- A10, 2011].

Detection of soluble enzymatic virulence factors:

Bacterial culture grown for 18 hours were evaluated for six different enzymatic virulence factors by cultivating the isolates on medium containing specific substrate for enzyme activity. All six enzymes and their specific medium are listed in following table: [Winstanley et al., 2005].

\begin{tabular}{|c|c|c|}
\hline Sr No & Name of Enzyme & Specific Medium \\
\hline 1 & Amylase & Starch Agar \\
\hline 2 & Caseinase & Skimmed Milk Agar \\
\hline 3 & Gelatinase & Gelatin Agar \\
\hline 4 & Haemolysins & Blood Agar \\
\hline 5 & Lecithinase & Egg Yolk Agar \\
\hline 6 & Lipase & Tributyrin Agar \\
\hline
\end{tabular}

\section{Detection of ESBL producers:}

All isolates were spread on Mueller- Hinton agar, Antibiotic disks Ceftazidime and ceftazidime/ clavulanic acid \& Cefotaxime and cefotaxime/ clavulanic acid discs were placed aseptically on the agar plate. A $15 \mathrm{~mm}$ distance was kept between two discs. Plates were incubated at 37c for 24 hours. The zone difference of $>5 \mathrm{~mm}$ was confirmed as ESBL Positive [CLSI Document M02- A10, 2011].

\section{Biofilm production}

All the isolates were subjected to detect their ability to form biofilm by two different methods: (1) Tube Method, (2) Congo red Agar Method [Christensen et al., 1985, Christensen et al., 1982, Freeman et al., 1989].

\section{Detection of T3SS genes}

Bacterial culture grown for 16 to 18 hours in nutrient broth was subjected for DNA isolation using commercial available kit. Genomic DNA was used as a template for PCR for four virulence genes listed in following table: [Holban et al., 2013].

\begin{tabular}{|c|c|c|}
\hline $\begin{array}{l}\text { Sr } \\
\text { no }\end{array}$ & Gene & Primer sequence (5'-3') \\
\hline 1 & exo $\mathrm{S}$ & $\begin{array}{l}\text { exo S -F(5'-GCG AGG TCA GCA GAG TAT CG-3') } \\
\text { exo S -R(5'-TTC GGC GTC ACT GTG GAT GC-3') }\end{array}$ \\
\hline 2 & exo $\mathrm{T}$ & $\begin{array}{l}\text { exo T-F(5'-AAT CGC CGT CCA ACT GCA TGC-3') } \\
\text { exo T -R(5'-TGT TCG CCG AGG TAC TGC TC -3') }\end{array}$ \\
\hline 3 & exo U & $\begin{array}{l}\text { exo U -F(5'-CCG TTG TGG TGC CGT TGA AG-3') } \\
\text { exo U -R(5'-CCA GAT GTT CAC CGA CTC GC-3') }\end{array}$ \\
\hline 4 & exo Y & $\begin{array}{l}\text { exo Y -F(5'-CGG ATT CTA TGG CAG GCA GG-3') } \\
\text { exo Y -R(5'-GCC CTT GAT GCA CTC GAC CA-3') }\end{array}$ \\
\hline
\end{tabular}

\section{Result and Discussion}

\section{Distribution of Pseudomonas aeruginosa isolates:}

As shown in the table a total of 125 clinical were identified. The highest number of isolates was from Urine 53(42.40\%), followed by Pus 26(20.80\%), Sputum 18 (14.40\%), Body fluids 14 (11.20\%), Wound 9(7.20\%), and Blood 5 (4\%). And 53 isolates were isolated from different vegetable and environment samples, where higher numbers was isolated from Parval $8(15.09 \%)$, followed by Carrot $4(7.55 \%)$, French beans $4(7.55 \%)$, and Tooriya $4(7.55 \%)$.

\begin{tabular}{|c|c|c|c|c|c|c|c|c|}
\hline \multicolumn{2}{|c|}{$\begin{array}{c}\text { Distribution of Pseudomonas aeruginosa } \\
\text { isolates from Clinical samples }\end{array}$} & \multicolumn{6}{|c|}{ Distribution of Pseudomonas aeruginosa isolates from environmental and vegetables } \\
samples
\end{tabular}

Isolation and Identification of Pseudomonas aeruginosa: Isolation and identification of Pseudomonas aeruginosa was carried out using standard microbiological procedures: Gram staining, Motility, colony morphology. This organism produces lactose non- fermenter yellow coloured colonies on MacConkey's agar, and on selective medium like King's B Medium base it produces characteristic blue- green pigment (Pyocyanin). The production of pyocyanin was observed in all the isolates. This virulence factor consists of blue- green phenazine pigment exclusively produced by Pseudomonas aeruginosa, which is related to tissue damage via formation of reactive hydroxyl radicals and superoxides. Positive
Oxidase test is a confirmatory test for identification of Pseudomonas aeruginosa. 


\section{International Journal of Science and Research (IJSR) \\ ISSN (Online): 2319-7064}

Index Copernicus Value (2015): 78.96 | Impact Factor (2015): 6.391
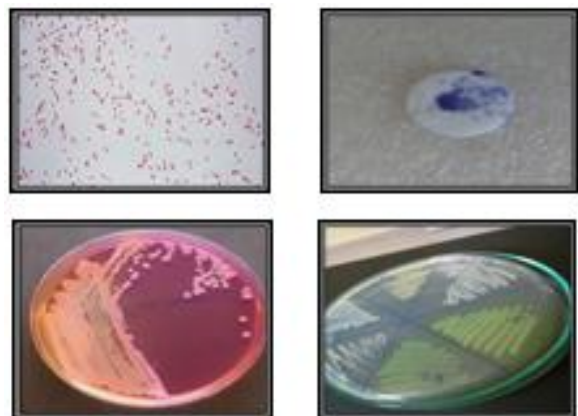

Figure 3: Identification of Pseudomonas aeruginosa; (A) Gram Staining, (B) Oxidase Test, (C) MacConkey's Agar,

(D) King's B Medium

\section{Antimicrobial Susceptibility Testing:}

The antimicrobial susceptibility profile obtained in this study showed that the most active drug against Pseudomonas aeruginosa was Amikacin (70.4\%), followed by Gentamicin (70.4\%), Netilin (69.6\%), and Tobramicin
(68\%). Pires et al reported in his study that the most active antimicrobials against Pseudomonas aeruginosa were Amikacin (84.6\%). And also isolates from environment and vegetables samples shows activity against Tobramicin $(100 \%)$ and Gentamicin $(98.11 \%)$

The results revealed high level of resistance to a significant number of antibiotics by Pseudomonas aeruginosa isolates. Overall $89.60 \%$ of isolates tested were resistant to Amoxyclav, $88 \%$ to Cefuroxime, $40.80 \%$ to Imipenem, and $36 \%$ to Meropenem. And all environment and vegetable isolates shows higher resistant to Amoxyclav 94\% and Cefuroxime $83.01 \%$.

The multidrug- resistant pattern was observed is consistent with reports of olayinka et al., 2004. This high level of resistance may be explained by the indiscriminate empirical use of broad spectrum antibiotics.

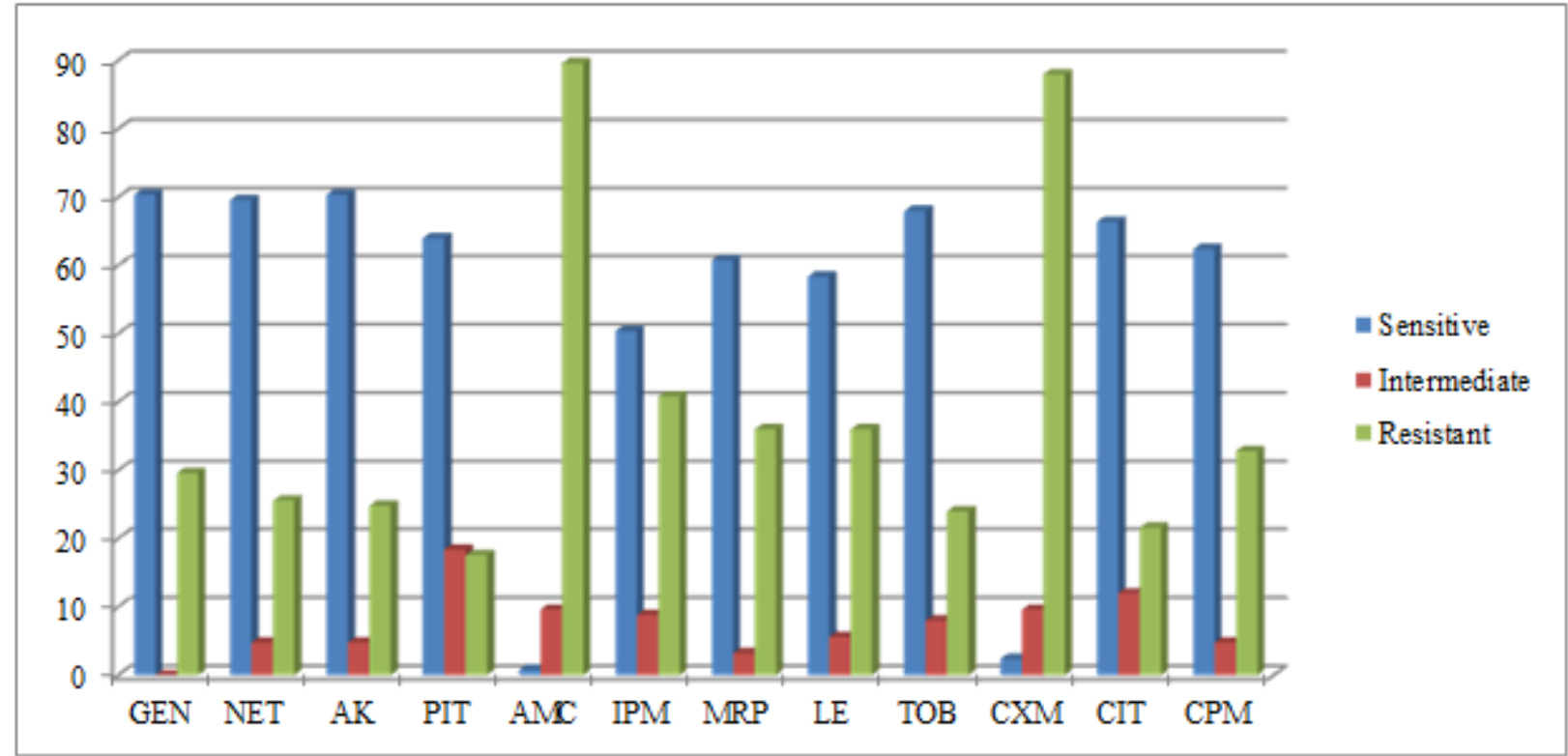

Figure 1: Antibiogram of Clinical isolates of Pseudomonas aeruginosa

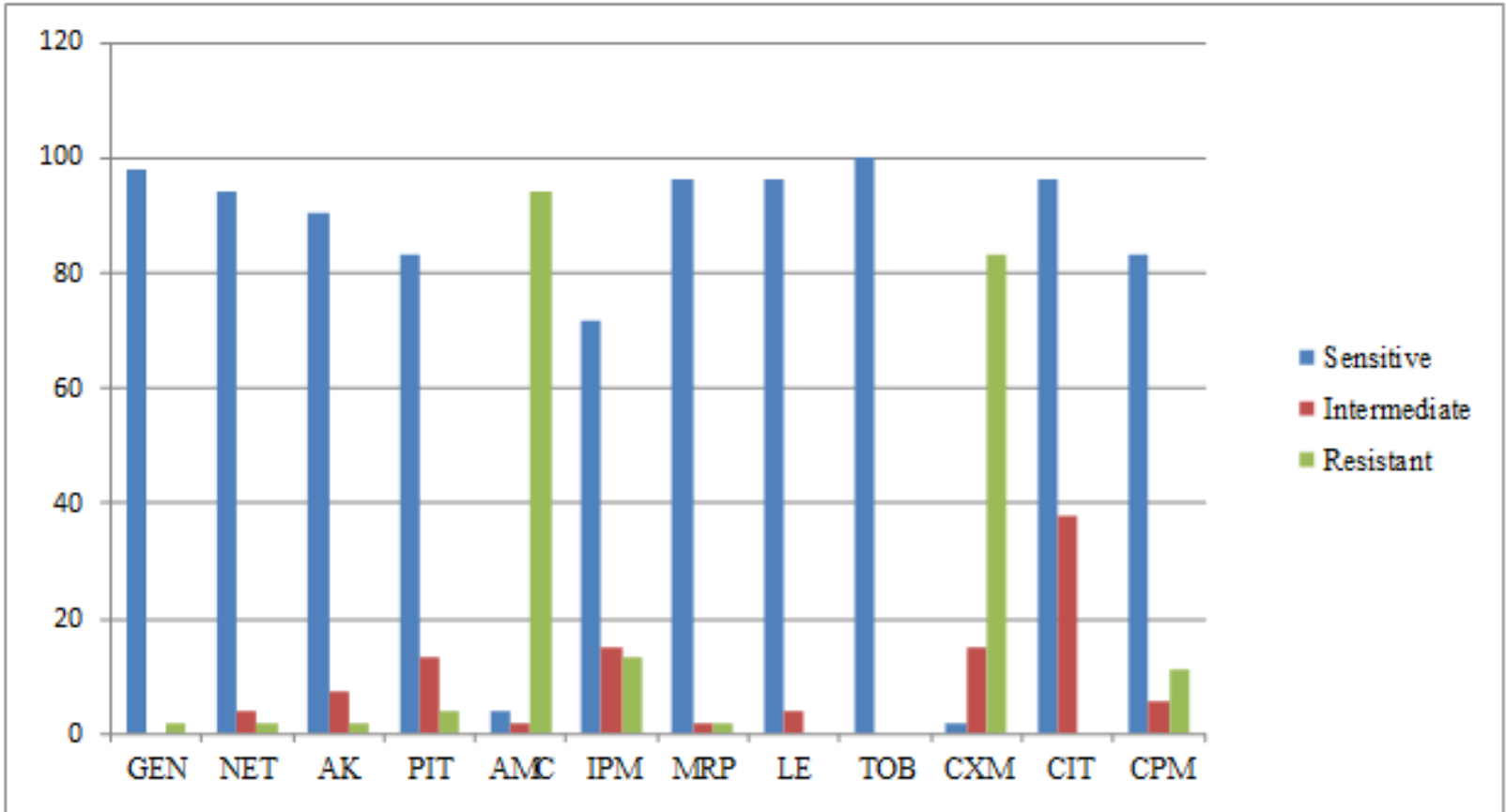

Figure 2: Antibiogram of Environment and vegetables isolates of Pseudomonas aeruginosa 


\section{International Journal of Science and Research (IJSR) \\ ISSN (Online): 2319-7064 \\ Index Copernicus Value (2015): 78.96 | Impact Factor (2015): 6.391}

Detection of soluble enzymatic factors:

In clinical isolates the production of Gelatinase $96.80 \%$, Lipase $96 \%$, Lecithinase $88 \%$, and Caseinase $88 \%$ corresponds to virulence factors associated with tissue injury. And in environment and vegetable isolates of Pseudomonas aeruginosa Gelatinase $100 \%$ and Lipase $100 \%$ were accounted,

\section{Detection of ESBL producers:}

Among 125 clinical isolates of Pseudomonas aeruginosa 65 $(52 \%)$ were ESBL producers and among 53 environment and vegetable isolates of Pseudomonas aeruginosa 25(47.17\%) were ESBL producers. ESBL producing Pseudomonas aeruginosa were isolated from Urine, pus and sputum. The highest ESBL producers were isolated from Urine samples. Our study reports similar results as by Nithyalaxmi (2016).

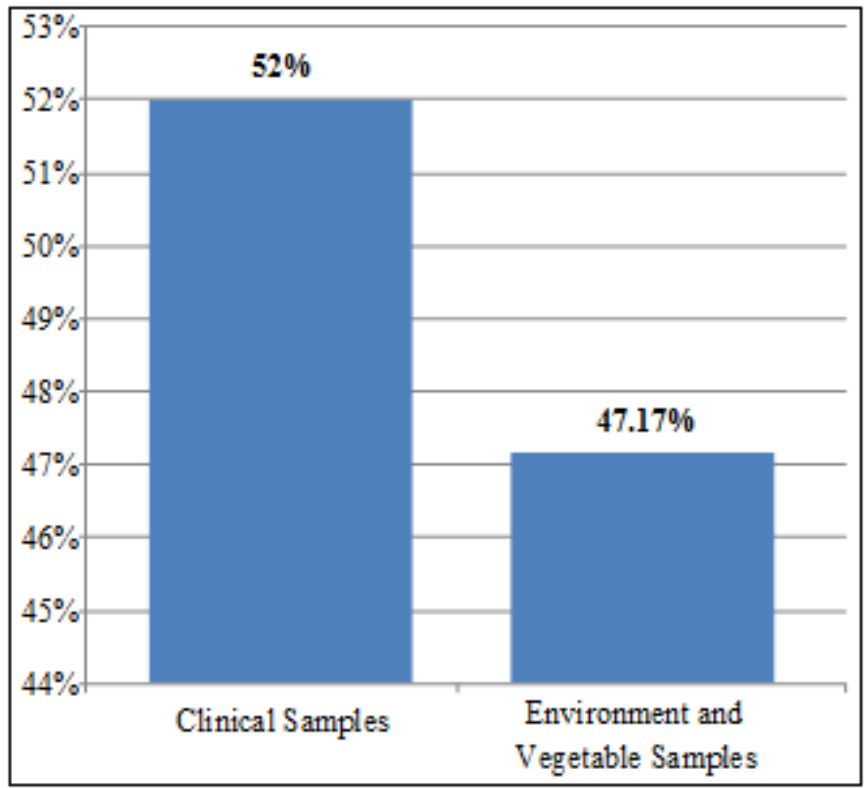

Figure 3: Results of ESBL producer isolates

\section{Biofilm Production}

Among 125 Clinical isolates, Tube method detected the number of strong biofilm producers were $36 \%$, moderate were $51.20 \%$, and non-biofilm producers were $12.8 \%$. And from 53 environment and vegetable isolates, this method detected the number of strong biofilm producers were $50.94 \%$, moderate were $41.51 \%$, and non-biofilm producers were $7.55 \%$. Among 125 Clinical isolates, Congo red agar method, detected the number of strong biofilm producers were $16.8 \%$, moderate were $52 \%$, and non-biofilm producers were $31.2 \%$. And from 53 environment and vegetable isolates, this method detected the number of strong biofilm producers were $13.2 \%$, moderate were $56.6 \%$, and nonbiofilm producers were $30.2 \%$. In our study we found that the majority of biofilm producing bacteria was from urine and pus. Similarly Donian reported the association of biofilm producing bacteria.

In all 178 isolates, Tube method detected $88.76 \%$ isolatesas biofilm producers and $11.23 \%$ as non- biofilm producers. And Congo red agar detected $69.10 \%$ as biofilm producers and $30.90 \%$ as non- biofilm producers. In another study, Ruzicka et al, noted that out of 147 isolates, TM detected biofilm formation in $79(53.7 \%)$ and CRA detected in 64 $(43.5 \%)$ isolates.

\section{Detection of T3SS genes}

The prevalence of type III secretion toxins- encoding genes detected by multiplex PCR in studied Pseudomonas aeruginosa isolates( $\mathrm{n}=25)$ were as follows(Given in below figure): exoU- $100 \%$, exoT- $96 \%$, exoS-92\% and exoY$92 \%$. The presence of the exoU gene was more commonly seen in all isolates suggest that it is an important factor in the development of acute invasive infections. Our study produces same results as Ciara and collaborators [65], who studied the contributions of T3SS genes as a virulence of Pseudomonas aeruginosa.

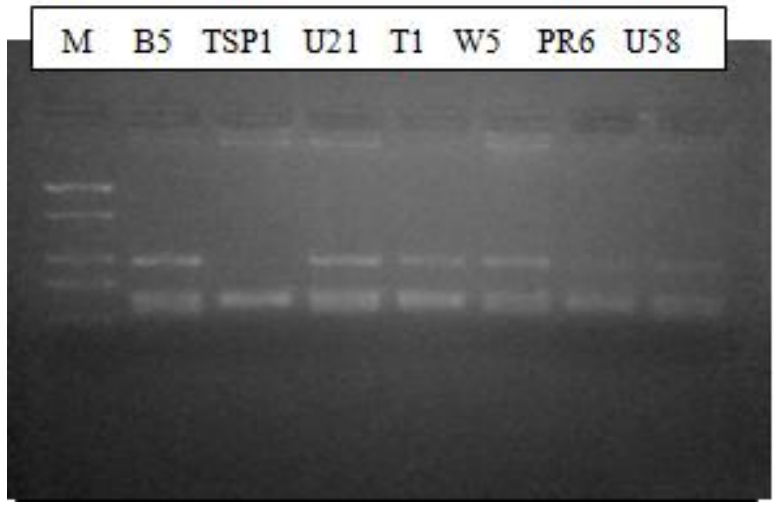

Figure: Results of T3SS genes

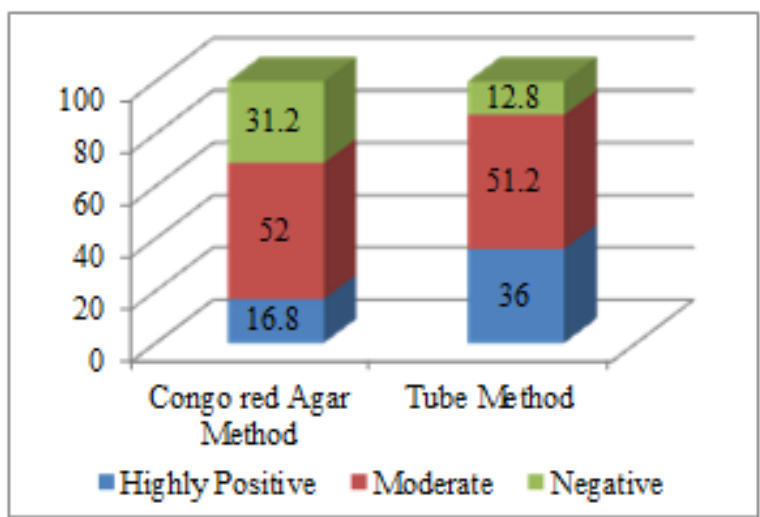

(i)

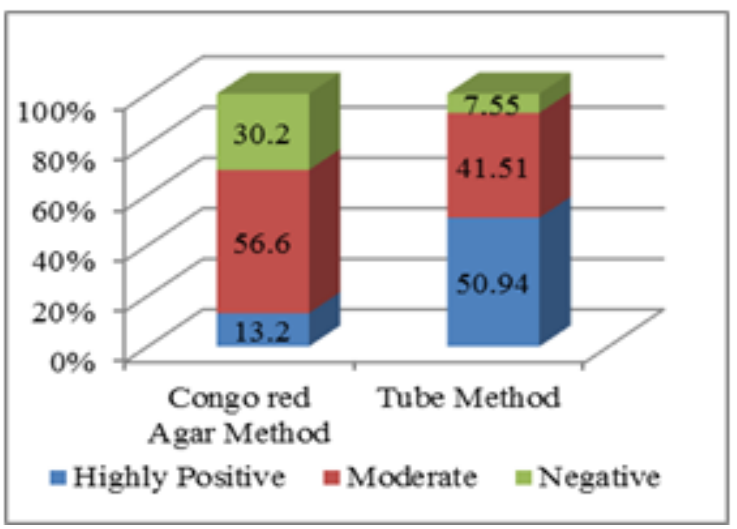

(ii)

Figure: (i) Results of biofilm formation of environment and vegetable isolates \& (ii) Results of biofilm formation of clinical isolates 


\section{International Journal of Science and Research (IJSR) \\ ISSN (Online): 2319-7064}

Index Copernicus Value (2015): 78.96 | Impact Factor (2015): 6.391

\begin{tabular}{|c|c|c|c|c|}
\hline \multirow{2}{*}{$\begin{array}{c}\text { Sources of } \\
\text { Isolates }\end{array}$} & \multicolumn{4}{|c|}{ Type 3 Secretion toxins- encoding genes } \\
\cline { 2 - 5 } & ExoS & ExoT & ExoU & ExoY \\
\hline Urine(15) & $15(100 \%)$ & $15(100 \%)$ & $15(100 \%)$ & $15(100 \%)$ \\
\hline Blood (3) & $3(100 \%)$ & $3(100 \%)$ & $3(100 \%)$ & $3(100 \%)$ \\
\hline Pus (2) & $2(100 \%)$ & $2(100 \%)$ & $2(100 \%)$ & $2(100 \%)$ \\
\hline Body Fluids (2) & $1(50 \%)$ & $1(50 \%)$ & $2(100 \%)$ & $1(50 \%)$ \\
\hline Wound (1) & $1(100 \%)$ & $1(100 \%)$ & $1(100 \%)$ & $1(100 \%)$ \\
\hline Tooriya (1) & $0(0 \%)$ & $1(100 \%)$ & $1(100 \%)$ & $0(0 \%)$ \\
\hline Parval (1) & $1(100 \%)$ & $1(100 \%)$ & $1(100 \%)$ & $1(100 \%)$ \\
\hline Total (25) & $23(92 \%)$ & $24(96 \%)$ & $25(100 \%)$ & $23(92 \%)$ \\
\hline
\end{tabular}

\section{Conclusion}

In conclusion, Pseudomonas aeruginosa isolates that cause infections at different sites exhibits variations in virulence profiles that reflects the organisms adaptive ability. Gentamicin and Amikacin were most susceptible antibiotics that can be used as the first drug of choice. The occurrence of multidrug resistant Pseudomonas aeruginosa has been serious implications on clinical prognosis of infections. Our results suggested that all Pseudomonas aeruginosa possesses some of the type III secretion genes. Therefore deep knowledge of the contribution of virulence factors their association with infection is require for development of the effective prevention and therapeutic procedures in patients with Pseudomonas aeruginosa infections.

\section{References}

[1] Shaver CM, Hauser AR.(2004) Relative contributions of Pseudomonas aeruginosa ExoU, ExoS, and ExoT to virulence in the lung. Infect Immun. Dec; 72 (12):6969-77.

[2] Galan JE and Collmer A. (1999) Type III Secretion machines: bacterial devices for protein delivery into host cells. Science, 284: 1322- 8 .

[3] Wong- Beringer A, Wiener- Kronish J, Lynch S, Flanagan J.(2008) Comparison of type III secretion system virulence among fluroquinolone- susceptible and- resistant clinical isolates of Pseudomonas aeruginosa. Clin Microbiol Infect; 14: 330-6.

[4] Deep A, Chaudhary U, and Gupta V, (2011) "Quorum sensing and bacterial pathogenicity: from molecules to disease," Journal of Laboratory Physicians, vol. 3, no. 1,pp. 4-11.

[5] Bjarnsholt T, (2013), "The role of bacterial biofilms in chronic infections," APMIS Supplementum, no.136, pp. 1-51.

[6] Todar K. (2009), The mechanisms of bacterial pathogenicity. Todar's online textbook of bacteriology.

[7] Veesenmeyer JL, Hauser AR, Lisboa T, Rello J. (2009). Pseudomonas aeruginosa virulence and therapy: evolving translational strategies. Crit Care Med; 37: 1777- 86.

[8] Hyun H, Chul K, Kim S, Hoe S. (2014), Correlation between virulence genotype and fluoroquinolone resistance in Carbapenem- resistant Pseudomonas aeruginosa.

[9] Engel JN. (2003) Molecular pathogenesis of acute Pseudomonas aeruginosa infections, In: Hauser AR, Rello J, eds. Severe infections caused by Pseudomonas

aeruginosa. Dordrech, Kluwer Academic Publishers: 201- 229.

[10] Lee VT, Smith RS, Tummler B, Lory D, (2005), Activities of Pseudomonas aeruginosa effectors secreted by the type III secretion system in vitro and during infection. Infect Immun ; 73: 1695- 1705.

[11] Vance RE, Rietsch A, mekalanos JJ, (2005), Role of the type III secreted exoenzymes $\mathrm{S}, \mathrm{T}$ and $\mathrm{Y}$ in systemic spread of Pseudomonas aeruginosa $\mathrm{Pa} 01$ in vivo. Infetc Immun ; 73: 1706- 1713.

[12] Lin HH, Huang SP, Teng HC, et al, (2006) Presence of exoU gene of Pseudomonas aeruginosa is correlated with cytotoxicity in MDCK cells but not with colonization in BALB mice. J Clin Microbiol; 44: 4595- 4597.

[13] Rasamiravaka T, Labtani q, Duez P, El jaziri M, (2015), The formation of biofilms by Pseudomonas aeruginosa: A review of the Natural and synthetic compounds interfering with control mechanisms. Biomed research international; 15: 1- 17.

[14] E. W. Koneman and G. D, Roberts, (1985) Journal of Basic Microbiology. 4SBN: 0-683-04746-9

[15] Bergey's manual of Determinative Bacteriology, $9^{\text {th }}$ edition, aand Jean F. Macfaddin, Biochemical tests for Identification of Medical Bacteria, $3^{\text {rd }}$ edition.

[16] Clinical and Laboratory Standards Institute (CLSI) (2011) Performance Standards for Antimicrobial Disk Susceptibility Tests; Approved Standard-Tenth Edition. CLSI Document M02-A10. Clinical and Laboratory Standards Institute, Wayne.

[17] Winstanley C, Kaye SB, Neal TJ, Chilton HJ, Miksch S, Hart CA; Microbiology Ophthalmic Group. Genotypic and phenotypic characteristics of Pseudomonas aeruginosa isolates associated with ulcerative keratitis. J Med Microbiol. 2005 Jun;54(Pt 6):519-26.

[18] Christensen, G. D., Simpson, W. A., Younger, J. J., Baddour, L. M., Barrett, F. F., Melton, D. M., \& Beachey, E. H. (1985). Adherence of coagulasenegative staphylococci to plastic tissue culture plates: a quantitative model for the adherence of staphylococci to medical devices. Journal of Clinical Microbiology, 22(6), 996-1006.

[19] Christensen GD, Simpson WA, Bisno AL, Beachey EH. (1982)Adherence of slime-producing strains of Staphylococcus epidermidis to smooth surfaces. Infect Immun.;37(1):318-26.

[20] Freeman DJ, Falkiner FR, Keane CT.(1989) New method for detecting slime production by coagulase negative staphylococci. J Clin Pathol.;42(8):872-4.

[21] Holban AM, Chifiriuc MC, Cotar AI, Bleotu C, Grumezescu AM, Banu O, et al. (2013) Virulence markers in Pseudomonas aeruginosa isolates from hospital acquired infections occurred in patients with underlying cardiovascular disease. Rom Biotechnol Letters.; 18: 8843-54.

[22] Olayinka AT, Olayinka BO, Onile, BA.(2009) Antibiotic susceptibility and plasmid pattern of Pseudomonas aeruginosa from the surgical unit of a university teaching hospital in north central Nigeria, International journal of medicine and medical sciences.; 079- 083 . 


\section{International Journal of Science and Research (IJSR) \\ ISSN (Online): 2319-7064}

Index Copernicus Value (2015): 78.96 | Impact Factor (2015): 6.391

[23] Nithayalaxmi J, Vidhyarani R, Mohanakridhnan K, Sumathi G,(2016) ESBL producing Pseudomonas aeruginosa in clinical

[24] specimens: Is is a scary nightmare or paper tiger?, Indian J Microbiol Res; 3(3): 287- 291.

[25] Donlan RM, Costerton JW. (2002) Biofilms: survival mechanisms of clinically relevant microorganisms. Clin Microbiol Rev. Apr;15(2):167-93.

[26] Růzicka F, Holá V, Votava M, Tejkalová R, Horvát R, Heroldová M, Woznicová V. (2004) Biofilm detection and the clinical significance of Staphylococcus epidermidis isolates. Folia Microbiol (Praha).;49(5):596-600. 